\title{
Évaluation préliminaire de la validité de construit d'un dispositif cartographique (carte procédurale) pour l'étude du raisonnement clinique
}

\author{
Preliminary evaluation of the construct validity of a new method \\ for clinical reasoning mapping
}

\author{
Pierre PotTiER ${ }^{1,2}$, Bernard Planchon ${ }^{1,2}$, Jean-Benoît HARdouin ${ }^{3}$, Véronique SEbILLE ${ }^{3}$, \\ Jean-Michel RoGEZ ${ }^{4}$ et Jacques-Henri BARRIER ${ }^{1,2}$ \\ 1 Service de médecine interne, Hôtel Dieu, Centre hospitalier universitaire de Nantes, Place Alexis Ricordeau, \\ 44093 Nantes Cedex 1, France \\ 2 Département de formation médicale continue et de développement pédagogique, Équipe de recherche nantaise en \\ éducation médicale (ERNEM), Faculté de Médecine de Nantes, 1 rue Gaston Veil, 44075 Nantes, France \\ 3 Laboratoire de biostatistique, Faculté de Pharmacie, Équipe d'accueil EA 4275, Recherche clinique et mesures \\ subjectives en sciences de la santé, Faculté de Médecine de Nantes, 1 rue Gaston Veil, 44075 Nantes, France \\ 4 Décanat, Faculté de médecine, 1 rue Gaston Veil, 44075 Nantes, France
}

Manuscrit reçu le 10 décembre 2008; commentaires éditoriaux formulés aux auteurs le 5 août 2009, le 17 janvier et le 31 mai 2011 ; accepté pour publication le $1^{\text {er }}$ juin 2011

\section{Mots clés :}

Stratégies de résolution de problème ; cartes conceptuelles ; raisonnement clinique ; technique de raisonnement à haute voix
Résumé - Contexte : L'étude du raisonnement clinique conduit à distinguer : 1) des stratégies de résolution de problème (SRP) inductives pendant lesquelles les hypothèses diagnostiques s'établissent à partir des faits et 2) des stratégies déductives reposant sur une collecte de signes orientée par la génération initiale d'hypothèses. Objectif : Explorer la viabilité d'un dispositif cartographique codifié, élaboré par des tiers observateurs, pour rendre compte de la structure des processus de raisonnement clinique développés en situation expérimentale, respectivement par des novices et par des experts. Méthode : Trois groupes de participants de niveaux d'expertise différents ont été constitués (étudiants en médecine $3^{\mathrm{e}}$ année ; $5^{\mathrm{e}}$ année et médecins internistes). Des représentations cartographiques apparentées aux cartes conceptuelles, dénommées cartes procédurales ont été transcrites par une tierce personne au fur et à mesure que les participants résolvaient à haute voix quatre problèmes complexes simulés par écrit. Cinq séquences unitaires de raisonnement préalablement définies (raisonnement non analytique, par résumé syndromique, par organigrammes décisionnels, catégoriel et déductif) ont été systématiquement recherchées et dénombrées sur les cartes. Leurs fréquences ont été établies en fonction du degré d'expertise et du type de problème. Résultats : Les séquences unitaires prédéterminées d'après les données de la littérature ont toutes été observées et répertoriées sur les cartes. Le raisonnement déductif a prédominé chez les experts (3,6 séquences inductives (SI) en moyenne par carte pour 6,2 séquences déductives). Les experts comme les novices ont utilisé des stratégies mixtes, inductives et déductives pour résoudre les problèmes 
Keywords:

Problem-solving strategies; concept mapping; clinical reasoning; reasoning out loud cliniques (taux de SI de 0,4 et 0,6). La fréquence de certaines séquences a varié en fonction de l'expertise et du type de dossier. Conclusion : Ce travail exploratoire montre que l'utilisation d'une méthode cartographique originale de représentation du raisonnement clinique retrouve les différentes SRP rapportées dans la littérature et suggère l'utilisation de stratégies mixtes chez les novices comme chez les experts, confrontés à des problèmes cliniques complexes simulés par écrit. Cet outil d'évaluation des processus impliqués dans le raisonnement clinique pourrait s'avérer utile pour dépister des dysfonctionnements chez des étudiants en difficulté.

Abstract - Context: The study of clinical reasoning implies distinguishing between inductive problem-solving strategies (PSS) that are forward-driven from facts and backward-driven problem-solving strategies, from hypotheses to facts. Objective: Exploring the construct validity of a new method for clinical-reasoning evaluation based on the reasoning-process structure mapping developed by a third party and experimented by novices and experts. Method: 3 groups of participants with various level of expertise (3rd- and 5th-year medical students and internists) were set up. An external individual drew up conceptual maps as participants reasoned out loud and solved 4 complex simulated clinical problems provided in writing. For each map, 5 predetermined problem-solving sequences (non-analytical reasoning, syndrome grouping, decision-making chart, scheme-inductive reasoning, category-inductive reasoning and deductive reasoning) were systematically searched and numbered. Frequencies were calculated according to the level of expertise and the type of clinical problem. Results: All predetermined problem-solving sequences were observed and mapped. Deductive reasoning was predominant among experts (a mean of 3.6 inductive sequences (IS) per map vs. 6.2 for deductive sequences). Both experts and novices used combined strategies (inductive and deductive) to solve clinical problems (IS rate ranged from 0.4 to 0.6 ). The frequency of some sequences varied according to the level of expertise and the type of problem. Conclusion: Usual problem-solving strategies were used through a new method of concept mapping. This study shows the predominance of mixed strategies both among novices and experts faced with complex clinical problems. The clinical-reasoning process assessment tool seems to be promising with respect to the screening of students with problem-solving difficulties.

\section{Introduction}

Le diagnostic est une étape importante du raisonnement clinique, participant au processus de la décision médicale ${ }^{[1]}$. En l'état actuel des connaissances scientifiques, la démarche diagnostique apparaît plutôt comme une compétence spécifique, liée à des contenus cognitifs et à un contexte d'application eux-mêmes spécifiques, que comme une compétence transversale polyvalente ${ }^{[2]}$. Il a été en effet démontré que savoir résoudre un problème clinique dans une situation donnée ne prédit pas la capacité à résoudre un problème similaire survenant dans un contexte différent, c'est-à-dire dans un autre domaine clinique ou dans le cadre d'une famille de situations différente. Établir un diagnostic à partir d'une situation médicale donnée constitue une activité de résolution de problème à part entière. Sur un plan général, en tant que processus, la résolution de problème nécessite deux étapes préalables que sont la position et la construction du problème, pouvant être réunies sous le terme générique de «problématisation ${ }^{[3]}$, séquence qui ne sera pas étudiée dans le cadre du présent travail. Plusieurs stratégies de résolution de problème ont été identifiées selon le sens de la démarche diagnostique entre les faits (données du problème) et les hypothèses émises ${ }^{[4]}$. La reconnaissance de forme ${ }^{[5,6]}$ et le raisonnement basé sur des schémas décisionnels préétablis ${ }^{[7]}$ sont des stratégies plutôt inductives caractérisées par le 
fait que les hypothèses diagnostiques s'établissent à partir des faits observés.

Le raisonnement hypothético-déductif ${ }^{[8]}$ comprend deux étapes : une étape de nature abductive (abduction : recherche d'un cadre théorique explicatif) où un ou plusieurs faits jugés pertinents par le clinicien déclenchent l'ouverture d'un cadre étiologique, encore appelé «catégorie », groupant une série d'hypothèses, et une étape déductive où chaque hypothèse précédemment émise est vérifiée en fonction des données observées - ou non - chez le patient et en référence à des connaissances antérieures. Les bases psychologiques de ce type de raisonnement sont en accord avec la théorie des scripts ${ }^{[9]}$, ces derniers désignant des types d'organisation de connaissances spécifiques, construits spécifiquement par les médecins au fur et à mesure de leur expérience, et décrivant sous forme de mini-scénarios des séquences attendues d'événements cliniques.

En pratique, le raisonnement clinique n'est jamais totalement inductif ou déductif ${ }^{[2,10,11]}$ ce qui rend sujet à caution les résultats d'études montrant une supériorité d'une stratégie par rapport à une autre ${ }^{[12]}$. Chez des experts, par exemple, il a été démontré qu'une procédure déductive de vérification des diagnostics différentiels fait souvent suite à une phase inductive initiale pendant laquelle une hypothèse principale est établie soit par reconnaissance de script soit après mise en œuvre d'un arbre décisionnel. Norman et al. ${ }^{[11]}$ ont montré que, dans des situations complexes, le raisonnement était mixte (inductif et déductif), tant chez les novices que chez les experts, mais que ce qui caractérisait le raisonnement expert était le nombre élevé d'inférences, qu'elles soient inductives ou déductives, et son efficience en terme de succès diagnostique.

D'autres études rapportent des recours à des stratégies variées de résolution de problème en fonction du niveau d'expertise et de la difficulté de la tâche. Les novices utiliseraient plus souvent le raisonnement hypothético-déductif tandis que la reconnaissance de forme serait l'apanage des experts ${ }^{[13,14]}$. Dans des situations diagnostiques difficiles en revanche, les raisonnements inductifs basés sur des organigrammes et les stratégies hypothéticodéductives seraient favorisés ${ }^{[4]}$.

S'il existe des outils quantitatifs d'évaluation du raisonnement clinique - visant à en évaluer le produit (tels que le test de concordance de script $\left.{ }^{[14,15]}\right)$-, les outils d'analyse qualitative visant à en décrire les processus - sont plus rares et font appel à des méthodes telles que les protocoles de pensée à voix haute (think aloud $)^{[5,11-13,16]}$, ou l'analyse structurale des cartes conceptuelles ${ }^{[17-19]}$. La principale difficulté en matière d'évaluation des stratégies du raisonnement clinique réside dans l'absence de gold standard, toutes les stratégies étant de fait acceptables dès lors que le raisonnement est pertinent et efficient. De ce fait, s'intéressant plus au produit, l'analyse basée sur l'échec ou la réussite n'apparait pas adaptée à l'évaluation des processus en jeu dans le raisonnement clinique, ce d'autant que la complexité inhérente aux problèmes médicaux est telle que plusieurs solutions peuvent parfois s'avérer possibles, en accord avec la conception admise d'un problème complexe ou mal structuré (ill structured problem ${ }^{[20]}$.

L'analyse de représentations figuratives du raisonnement clinique, bien que séduisante en tant que méthode de recherche dans le domaine du raisonnement clinique, a été surtout utilisée comme outil d'évaluation des acquis lors de séances d'apprentissage ${ }^{[21-23]}$. Parmi ces représentations, celles élaborées grâce à la mise en œuvre de la technique de cartographie conceptuelle a été le plus souvent utilisée bien que d'autres modes de représentation graphique des activités mentales existent. Une carte conceptuelle se définit par la figuration de concepts, des liens - de nature polymorphe - qui les relient les uns aux autres et de la hiérarchisation de ces concepts (notions de concepts subordonnés ou super-ordonnés) ${ }^{[24]}$. Nous postulons que l'élaboration instantanée d'une représentation figurative cartographique des activités cognitives mises en œuvre pendant le raisonnement diagnostique pourrait constituer un outil particulièrement intéressant pour reconnaître les stratégies de résolution de problème (inductives ou déductives). Nous suggérons 
de dénommer ces représentations par le terme de « cartes procédurales » pour les distinguer des cartes conceptuelles dont elles diffèrent quelque peu.

L'objectif principal de ce travail exploratoire était d'explorer la viabilité d'un dispositif cartographique codifié, élaboré par des tiers observateurs, pour rendre compte de la structure des processus de raisonnement clinique développés en situation expérimentale, respectivement par des novices et par des experts. L'objectif secondaire était de décrire les variations des séquences unitaires préalablement modélisées en fonction du niveau d'expertise et du type de problème clinique.

\section{Méthodes}

\section{Modélisation des séquences de raisonnement}

Comme l'a bien montré Eva ${ }^{[2]}$, un raisonnement diagnostique est le plus souvent basé sur des stratégies de résolution mixtes, analytiques et non analytiques, inductives et déductives, avec des passages itératifs d'un mode à l'autre. Nous utiliserons le terme «séquence » pour dénommer une partie du raisonnement correspondant à un mode de résolution bien identifié dans la littérature. Il est ainsi possible de modéliser des séquences inductives (définies par un raisonnement guidé des faits vers les hypothèses) et des séquences déductives (définies par un raisonnement conduit à partir des hypothèses vers les faits). Pour ce travail, les séquences identifiées combinent à la fois le type de stratégie de résolution de problème mise en œuvre et les représentations cognitives activées et sur lesquelles le processus s'appuie. La reconnaissance de forme (de nature inductive) et la vérification a posteriori de la probabilité d'un diagnostic par la référence à des faits présents ou absents (de nature déductive) sont des stratégies dynamiques de raisonnement bien documentées. Les représentations cognitives sont des modalités plus ou moins compilées de stockage de l'information en mémoire. Elles peuvent être appelées au cours du raisonnement tant dans une phase inductive que déductive. Il s'agit des scripts cliniques, des catégories, des regroupements syndromiques et des organigrammes décisionnels

D'après les données de la littérature, nous avons identifié cinq séquences distinctes (cf. figure 1) :

1) la reconnaissance de forme (pattern recognition) génère de façon inductive, après intégration d'un nombre variable de faits, un diagnostic final à partir de l'activation soit d'un script (miniscenario clinique), soit d'un prototype (moyenne représentative de toutes les situations cliniques antérieurement rencontrées), soit d'un exemple concret stocké en mémoire à long terme (instance),

2) l'utilisation d'un regroupement syndromique conduit à générer, à partir de plusieurs faits, une seule hypothèse intermédiaire, utilisée par la suite, en association soit à d'autres hypothèses générées ou non de façon identique, soit à d'autres faits,

3) l'utilisation de catégories permet une génération de plusieurs hypothèses d'emblée (au moins deux) à partir d'un ou plusieurs faits ou d'une hypothèse préétablie,

4) l'utilisation d'organigrammes décisionnels combine une hypothèse préalablement générée et un fait pour induire une seconde hypothèse intermédiaire,

5) les séquences de vérification d'hypothèses consistent à justifier ou réfuter ces dernières, en référence à un ou plusieurs faits recherchés a posteriori, en lien avec ces hypothèses émises.

\section{Construction des problèmes cliniques simulés par écrit (cf. annexe)}

Quatre problèmes ont été construits par deux médecins internistes en faisant varier la présentation clinique :

- dans le problème clinique $n^{\circ} 1$, tous les signes nécessaires et suffisants au diagnostic étaient d'emblée présents pour permettre un diagnostic instantané par reconnaissance de forme tel que décrit par Norman et al. ${ }^{[6]}$; 


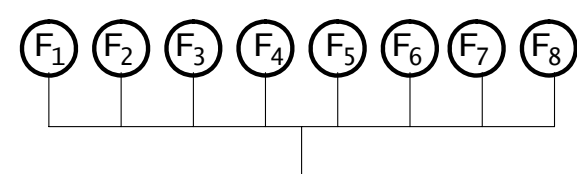

Séquence non analytique

de type reconnaissance de forme

$\mathrm{H}_{1}$

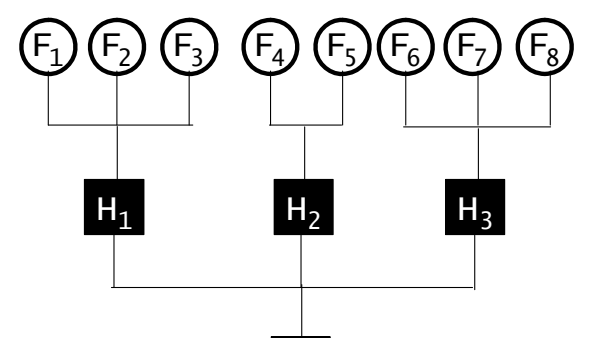

$\mathrm{H}_{4}$

Séquence de type regroupement syndromique

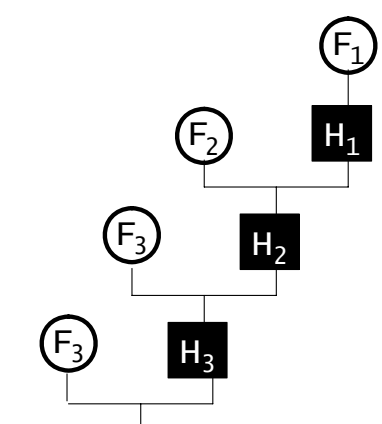

$\mathrm{H}_{4}$

Séquence de type organigrammes décisionnels

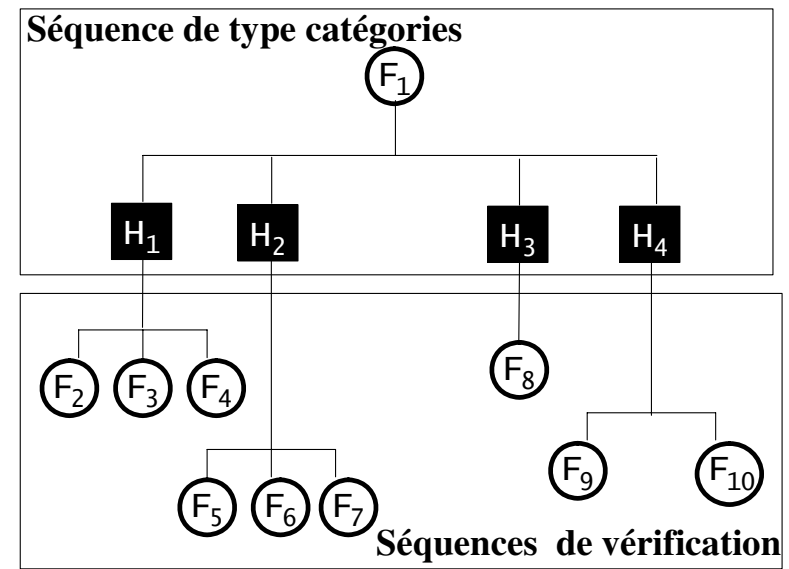

\section{Stratégies moins élaborées}

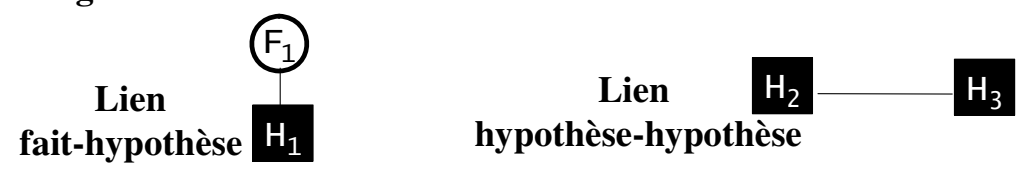

Fig. 1. Modélisation des séquences de raisonnement diagnostique. Fi : fait (concepts apparaissant dans l'énoncé du problème) utilisé en position i dans le raisonnement. Hi : hypothèse (concept émis n'apparaissant pas dans l'énoncé) générée en position i dans le raisonnement.

- dans le problème clinique $\mathrm{n}^{\circ} 2$, tous les signes utiles au diagnostic étaient également présents mais, en raison du caractère multisystémique de cette affection, la solution pouvait apparaître plus facilement en procédant à des regroupements syndromiques ;
- dans le problème clinique $n^{\circ} 3$, plusieurs diagnostics restaient plausibles à la fin de l'analyse tandis qu'un certain nombre d'autres pouvaient éventuellement être exclus grâce à l'existence de signes discriminants en fonction d'un arbre décisionnel préétabli ; 
- dans le problème clinique $\mathrm{n}^{\circ} 4$, en accord avec Heemskerk et al. ${ }^{[5]}$, nous avons postulé que la difficulté de la tâche diagnostique, liée à la multiplicité des hypothèses possibles était susceptible d'induire un raisonnement hypothético-déductif tel que décrit plus haut.

\section{Groupes constitués}

Trois groupes de quatre participants volontaires de niveaux d'expertise diagnostique différents ont été constitués (étudiants en troisième année de médecine, étudiants en cinquième année de médecine et médecins internistes qualifiés). Les étudiants en troisième et cinquième année de médecine ont été sélectionnés au hasard.

Les étudiants en troisième année de médecine avaient été antérieurement confrontés aux différents thèmes abordés par les quatre problèmes cliniques, essentiellement sous l'angle de l'apprentissage de la sémiologie.

Les étudiants en cinquième année de médecine étaient censés connaître la pathologie de chacun des thèmes abordés, tant sur un plan pratique (au cours des stages hospitaliers) que sur un plan théorique (programme des cours dispensés à la faculté).

\section{Élaboration des cartes}

Notre méthode de cartographie doit être distinguée de celle produisant des cartes conceptuelles ${ }^{[24]}$ qui admettent une définition précise rappelée en introduction.

Les points communs entre les deux méthodes sont :

- la représentation de concepts et non pas de phrases ou de périphrases comme dans les cartes mentales par exemple;

- l'absence de limitation du champ des concepts, qu'ils s'agissent des hypothèses ou des faits (les participants étaient informés qu'ils pouvaient utiliser des faits absents des énoncés des problèmes pour argumenter leurs hypothèses).
Nos cartes procédurales divergent cependant nettement des cartes conceptuelles sur plusieurs points :

- les liens entre les concepts ne sont pas polymorphes mais binaires (inductifs ou déductifs) de nature uniquement causale comme dans les cartes dites cognitives (mais dans ces dernières, l'emploi de périphrase est privilégiée par rapport aux concepts) ;

- aucune hiérarchisation dans les concepts n'est demandée alors qu'il s'agit d'une caractéristique obligatoire des cartes conceptuelles ;

- dans une perspective d'évaluation des compétences, les cartes conceptuelles sont souvent élaborées sur une longue période par des retouches successives. À l'inverse, le caractère instantané de nos représentations, décrivant une succession chronologique d'activités mentales (les concepts et les faits sont numérotés par ordre d'apparition et toutes les séquences ne sont pas forcément reliées entre elles) en fait une méthode originale qui justifie la dénomination de cartes procédurales.

Les cartes ont été rédigées par une même tierce personne observatrice, à partir du raisonnement exprimé à voix haute par le participant. Aucune contrainte de temps n'a été imposée. Le rédacteur pouvait à tout moment interrompre le participant pour lui demander de clarifier son raisonnement si nécessaire. Les cas ont été soumis au participant les uns après les autres. La consigne consistait à résoudre le problème clinique en précisant que plusieurs diagnostics pouvaient rester plausibles à la fin de l'analyse, que l'obtention d'un diagnostic de certitude n'était pas obligatoire et que les faits non rapportés dans les énoncés devaient être considérés comme absents.

Les faits ont été représentés par des cercles et étaient définis par tous concepts apparaissant dans l'énoncé du problème. Les hypothèses ont été représentées par des carrés et étaient définis par tout concept n'apparaissant pas dans l'énoncé. Les faits et les hypothèses ont été numérotés dans l'ordre de leur utilisation et d'apparition au cours du raisonnement. Les liens reliant les faits et les hypothèses 


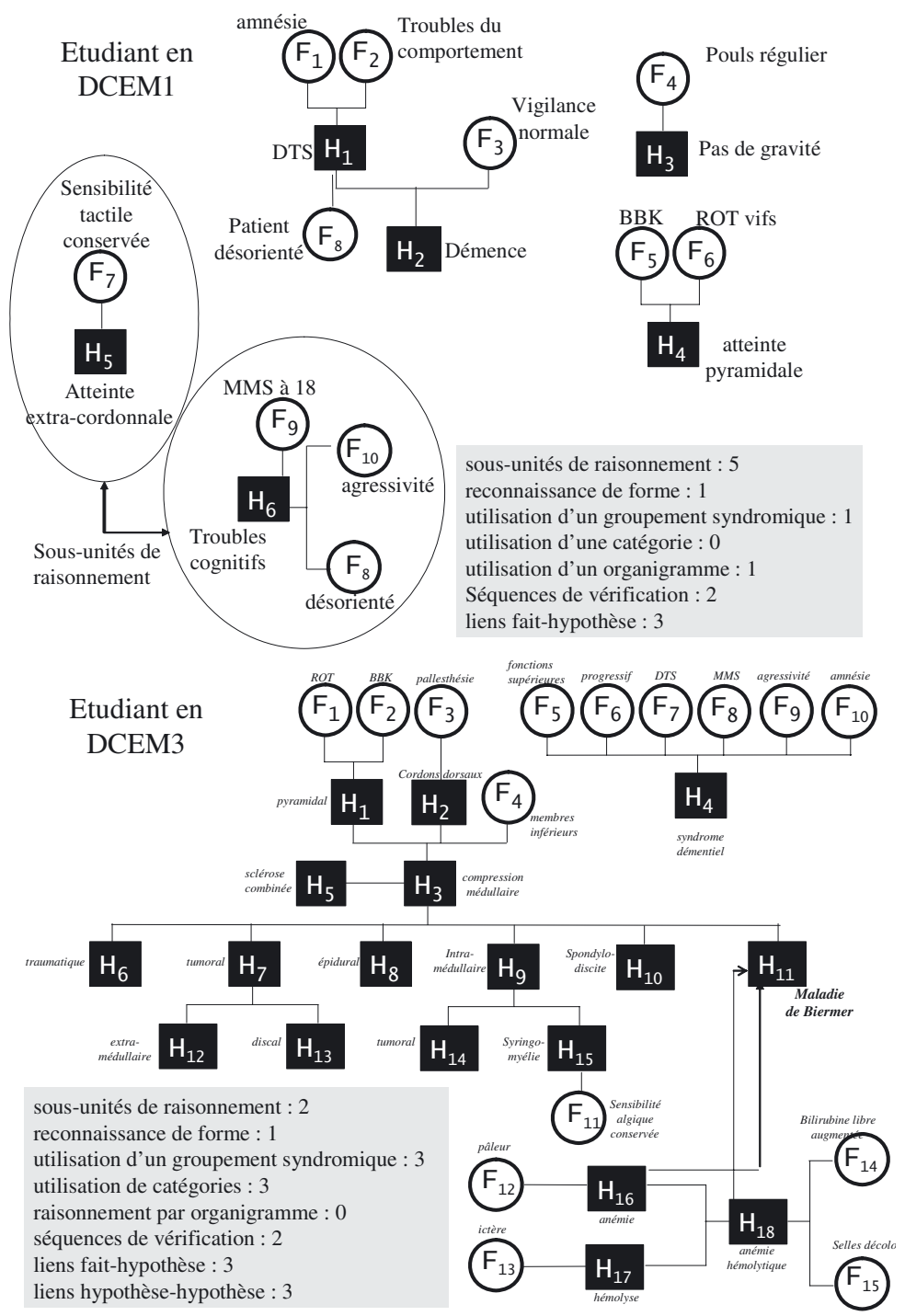

Fig. 2. Exemples de cartes procédurales et de leur analyse structurelle. DTS : désorientation temporo-spatiale. BBK : Réflexe cutané plantaire en extension (signe de Babinski). MMS : mini-mental status. ROT : réflexes ostéo-tendineux. Fi : fait (concepts apparaissant dans l'énoncé du problème) utilisé en position i dans le raisonnement. Hi : hypothèse (concept émis n'apparaissant pas dans l'énoncé) générée en position i dans le raisonnement. DCEM1 : première année du deuxième cycle des études médicales.

étaient représentés en rouge s'il s'agissait de liens inductifs ou en vert pour les liens déductifs.

\section{Analyse des cartes (cf. figures 1 et 2).}

L'analyse des 48 cartes construites (trois groupes de quatre participants, soumis chacun à quatre situations) a suivi un protocole quantitatif. En effet, les structures de raisonnement prédéterminées ont été repérées puis dénombrées.

Ainsi plusieurs paramètres ont été répertoriés :

1) le nombre de séquences, totalement indépendantes, non reliées entre elles, appelées sousunités de raisonnement ; 
2) des séquences faisant appel à la reconnaissance d'une représentation cognitive préalablement mémorisée. Parmi ces représentations cognitives, nous avons individualisé :

- les séquences de type "reconnaissance de forme », repérées lorsque plusieurs faits conduisaient à un seul diagnostic, ce diagnostic étant final c'est-à-dire qu'il n'était plus utilisé par la suite dans le raisonnement ;

- les groupements syndromiques, repérés lorsque plusieurs symptômes étaient associés pour former une hypothèse qui était utilisée par la suite dans le raisonnement ;

- les catégories, identifiées lorsque des listes d'hypothèses étaient reliées à un symptôme par des liens physiopathologiques ou purement mnémotechniques. Selon nos règles de représentation graphique, une catégorie était repérée lorsqu'un seul fait était relié à plusieurs hypothèses ;

- les organigrammes décisionnels, arbres diagnostiques basés sur des symptômes discriminants permettant une orientation binaire du diagnostic, reconnus lorsqu'un fait était associé à une hypothèse pour générer une seconde hypothèse.

$\mathrm{Si}$, en théorie, ces représentations cognitives peuvent être activées lors d'un raisonnement inductif ou déductif, la reconnaissance de forme, le groupement syndromique et les organigrammes décisionnels sont le plus souvent associés au raisonnement inductif tandis que l'utilisation de catégories est le plus souvent associée au raisonnement déductif. Pour chacune de ces séquences, nous avons identifié le mode raisonnement (inductif ou déductif) auquel elles étaient associées en fonction du sens des liens entre les faits et les hypothèses.

3) Les séquences de vérification d'hypothèse (toujours de nature déductive) correspondaient à la justification d'une hypothèse antérieurement émise par un ou plusieurs faits mentionnés (justification positive) ou non mentionnés (justification négative) dans l'énoncé.
4) Les liens n'entrant pas dans les séquences décrites ci-dessus (appelés liens non structurés) correspondaient aux liens entre un seul fait et une seule hypothèse (le plus souvent nature inductive) et les liens entre deux hypothèses (de nature déductive).

\section{Analyse statistique}

Les fréquences des différentes séquences définies et les moyennes des séquences inductives et déductives ont été calculées. Aucune comparaison statistique n'a été effectuée compte tenu du faible effectif dans chaque sous-groupe et du caractère exploratoire de ce travail.

\section{Résultats}

\section{Données globales}

Quarante-huit cartes (4 participants dans chaque groupe) ont été élaborées par le même investigateur, soit 16 cartes par niveau d'expertise ou encore 12 cartes par problème. À titre d'exemple, deux cartes sont représentées en figure 2.

Les fréquences des séquences de raisonnement clinique en fonction de leur nombre décompté sur les cartes sont représentées en figure 3 .

Toutes les séquences de raisonnement préalablement déterminées ont été observées sur les cartes quels que soient le niveau et le type de dossier. Certaines cartes comprenaient jusqu'à cinq sous-unités de raisonnement. Les organigrammes décisionnels ont été globalement moins utilisés que les autres représentations cognitives (catégories et groupement syndromique).

Le raisonnement inductif était principalement composé de séquences de reconnaissance de forme, de regroupement syndromique et d'organigramme décisionnels. Le raisonnement déductif s'est développé principalement grâce à l'utilisation de catégories à partir desquelles des séquences de vérification d'hypothèses étaient initiées. 


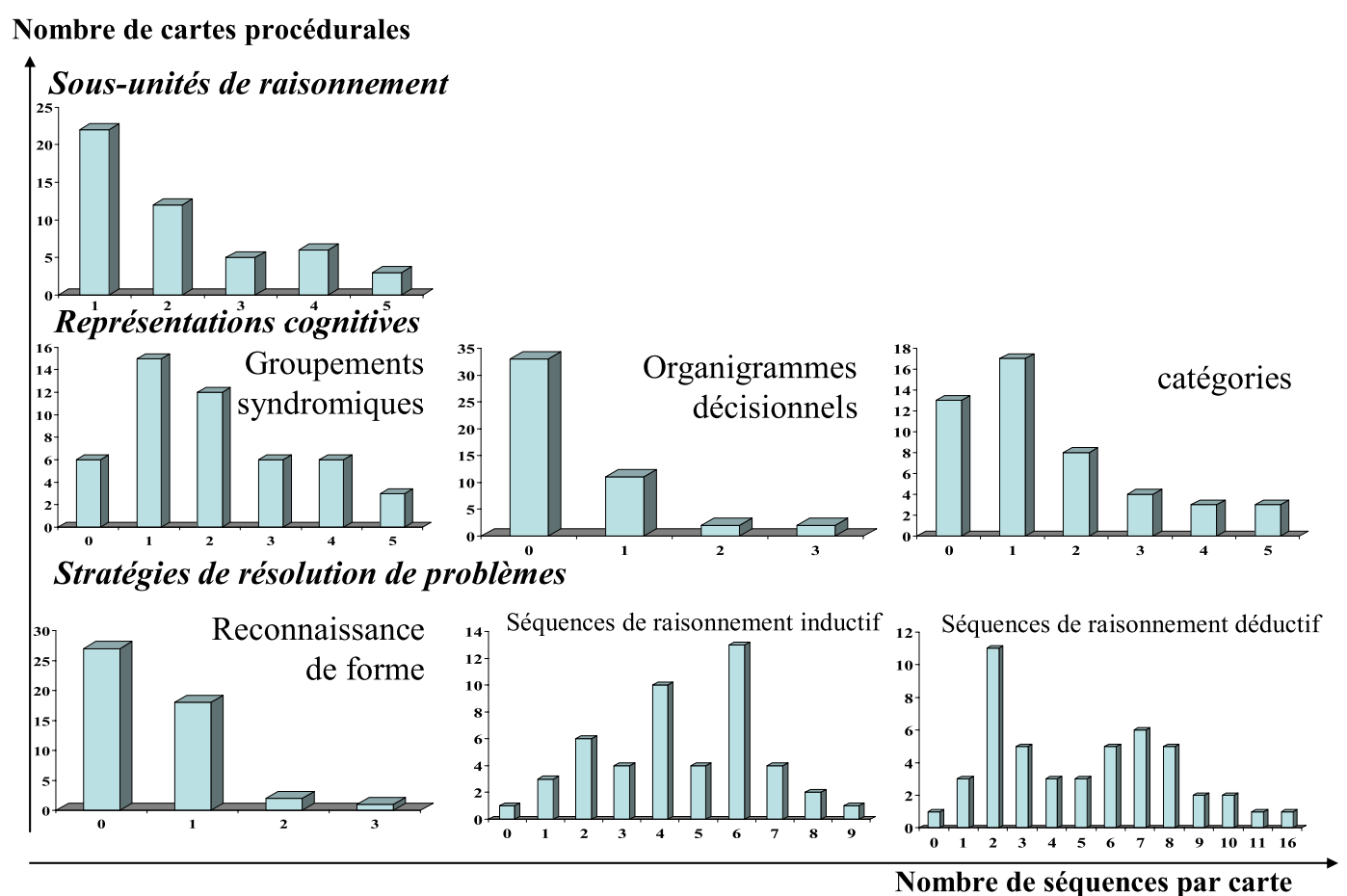

Fig. 3. Fréquences des séquences de raisonnement représentés sur des cartes procédurables réalisées par un tiers pendant le raisonnement à haute voix des participants.

Tous problèmes cliniques et tous niveaux confondus, le raisonnement mixte a prédominé. En moyenne 5,1 séquences inductives ont été retrouvées par carte pour 4,1 séquences déductives, avec un taux global de séquences inductives à $50 \%$ (cf. tableau I).

\section{Analyse selon les groupes}

\section{Analyse selon le niveau d'expertise (cf. tableau I)}

Le nombre moyen d'unités de raisonnement était de 1,4 pour les experts et 2,4 pour les novices. Le raisonnement catégoriel semble moins utilisé par les novices (1,2 vs. 1,9 pour les internistes), les liens non structurés apparaissent plus fréquents chez les étudiants en troisième année (2,2 vs.1,2 pour les étudiants de cinquième année et les internistes). Les internistes ont eu plus souvent recours au raisonnement déductif que les étudiants (taux de séquences inductives à $40 \%$ vs. $60 \%$ ) (cf. tableau I).

\section{Analyse selon le type de problème clinique (cf. tableau I)}

Le problème clinique $\mathrm{n}^{\circ} 1$ a très peu induit de raisonnement catégoriel ( 0,6 séquence par carte en moyenne). Le raisonnement syndromique a été plus souvent appliqué pour résoudre le problème clinique $n^{\circ} 2$ (3,3 groupements syndromiques par carte en moyenne). Ce problème a d'ailleurs été celui qui a déclenché le plus de séquences inductives (6,2 séquences par carte). Le problème $\mathrm{n}^{\circ} 3$ a généré le plus de séquences déductives ( 6,2 par carte). Le problème $\mathrm{n}^{\circ} 4$ est cependant celui qui est solutionné avec le taux de raisonnement déductif le plus fort $(70 \%)$.

\section{Discussion}

L'objectif principal de ce travail exploratoire était d'explorer la viabilité d'un dispositif cartographique codifié, élaboré par des tiers observateurs, pour rendre compte de la structure des processus de 
Tableau I. Moyennes des stratégies de raisonnement selon le niveau d'expertise et selon le problème clinique.

\begin{tabular}{cccccccccc} 
& \multicolumn{3}{c}{$\begin{array}{c}\text { NIVEAU D'EXPERTISE } \\
N=16\end{array}$} & \multicolumn{5}{c}{ PROBLEMES CLINIQUES } \\
& $\begin{array}{c}\text { Étudiant } \\
\text { de } \mathbf{3}^{\mathrm{e}} \text { année \# }\end{array}$ & $\begin{array}{c}\text { Étudiant } \\
\text { de 5e } \mathbf{5}^{\mathrm{e}} \text { année \# }\end{array}$ & Internistes & $\mathbf{1}$ & $\mathbf{2}$ & $\mathbf{3}$ & $\mathbf{4}$ & $\begin{array}{c}\text { moyennes } \\
\text { générales }\end{array}$ \\
\hline $\begin{array}{c}\text { Raisonnement } \\
\text { inductif* }\end{array}$ & 5,0 & 5,0 & 3,6 & 4,7 & 6,2 & 4,8 & 2,3 & 5,1 \\
\hline $\begin{array}{c}\text { Raisonnement } \\
\text { déductif** }\end{array}$ & 4,2 & 4,8 & 6,2 & 3,2 & 5,4 & 6,2 & 5,4 & 4,5 \\
\hline Taux d'induction $\S$ & 0,6 & 0,5 & 0,4 & 0,6 & 0,6 & 0,4 & 0,3 & 0,5 \\
\hline
\end{tabular}

* Raisonnement allant des faits vers les hypothèses faisant appel le plus souvent à des représentations cognitives du type script, groupement syndromique ou organigrammes décisionnels. ** Raisonnement allant des hypothèses vers les faits faisant appel le plus souvent à des représentations cognitives de type catégories. § Moyenne des séquences inductives sur la totalité des séquences inductives et déductives. \# $3^{\mathrm{e}}$ et $5^{\mathrm{e}}$ année des études médicales.

raisonnement clinique développés en situation expérimentale, respectivement par des novices et par des experts. La complexité était liée au caractère pluridisciplinaire des thématiques abordées et au fait que, comme le laissait entendre la consigne donnée aux participants, plusieurs hypothèses pouvaient rester plausibles au terme de l'analyse.

Le principal apport de ce travail est de montrer que les différentes stratégies de résolution de problème rapportées dans la littérature sont identifiables par l'analyse structurelle des représentations cartographiques codifiées élaborées par un tiers observateur, ce qui apporte des arguments en faveur de la validité de construit du dispositif cartographique élaboré.

La représentation cartographique instantanée du processus de raisonnement clinique et son élaboration par un tiers sont deux innovations potentiellement utiles pour le «diagnostic» de problèmes de raisonnement chez des médecins ou des étudiants en difficulté. En effet, les protocoles de «pensée à voix haute » sont habituellement analysés de façon différée à partir de la transcription de l'enregistrement des verbatims, ce qui ne permet pas de porter un diagnostic immédiat sur la qualité du raisonnement diagnostique du sujet. Par ailleurs, l'élaboration de la représentation cartographique par le participant lui-même, peut conduire à des résultats discutables liés à la difficulté de résoudre un problème et de construire une carte en même temps.

L'objectif secondaire du travail était par ailleurs de décrire les variations des séquences unitaires préalablement modélisées en fonction du niveau d'expertise et du type de problème clinique. À l'instar des travaux de Norman et d'Eva ${ }^{[2]}$, ce travail montre que, dans cette situation non authentique de questionnement diagnostique, les stratégies de résolution de problème utilisées sont mixtes. Certaines séquences inductives apparaissent plus souvent utilisées que d'autres, tel que le regroupement syndromique. L'utilisation du raisonnement catégoriel constitue la base des séquences déductives. La reconnaissance de script et le raisonnement basé sur les organigrammes apparaissent comme des stratégies plus rarement mises œuvre. Cette étude exploratoire ne permet pas de savoir si ces différences sont liées à la supériorité éprouvée ou ressentie d'une stratégie par rapport à une autre et si elles sont liées aux modalités d'apprentissage ou d'organisation des connaissances ou encore au niveau d'expertise. On peut cependant penser, même si cela reste à démontrer, qu'une mémorisation «syndromique » des données conduira préférentiellement à l'utilisation des stratégies de résolution de type syndromique, tout comme une mémorisation d'organigrammes décisionnels sera de nature à favoriser la résolution de problème en utilisant des signes discriminants. 
Tableau II. Proportions relatives de raisonnement inductif et déductif selon le niveau d'expertise d'après les données de la littérature et celles de l'étude.

\begin{tabular}{|c|c|c|c|c|}
\hline $\begin{array}{c}\text { Participants classés par } \\
\text { niveau croissant d'expertise }\end{array}$ & $\begin{array}{l}\text { Méthode d'évaluation du } \\
\text { raisonnement clinique }\end{array}$ & Tâche diagnostique & TRI & TRD \\
\hline Étudiant en $3^{\mathrm{e}}$ année & $\begin{array}{l}\text { Penser à voix haute } \\
\qquad+ \\
\text { cartes conceptuelles }\end{array}$ & $\begin{array}{c}4 \text { problèmes cliniques complexes } \\
\text { sur des thématiques différentes } \\
\text { simulés par écrit }\end{array}$ & $60 \%$ & $40 \%$ \\
\hline Étudiant en $5^{\mathrm{e}}$ année & & Idem & $50 \%$ & $50 \%$ \\
\hline Étudiant en $6^{\mathrm{e}}$ année ${ }^{[12]}$ & $\begin{array}{l}\text { Penser à voix haute } \\
\qquad+ \\
\text { analyse instantanée }\end{array}$ & $\begin{array}{c}4 \text { problèmes cliniques de } \\
\text { gastro-entérologie non complexes } \\
\text { (un seul diagnostic possible) }\end{array}$ & $41 \%$ & $59 \%$ \\
\hline $\begin{array}{c}\text { Internes en } 1^{\mathrm{e}} \text { année de } 3^{\mathrm{e}} \text { cycle } \\
\text { de médecine générale }\end{array}$ & $\begin{array}{c}\text { Penser à voix haute } \\
+\end{array}$ & $\begin{array}{c}\text { Résoudre } 11 \text { problèmes } \\
\text { cliniques complexes }\end{array}$ & $64 \%$ & $36 \%$ \\
\hline $\begin{array}{l}\text { Internes en } 1^{\mathrm{e}} \text { et } 2^{\mathrm{e}} \text { année } \\
\text { de DES de médecine } \\
\end{array}$ & $\begin{array}{l}\text { analyse différée } \\
\text { sur les verbatims }\end{array}$ & Idem & $62 \%$ & $38 \%$ \\
\hline $\begin{array}{c}\text { Internes en DES } \\
\text { de médecine interne }{ }^{[4]}\end{array}$ & enregistrés & QROC ou QCM longue liste & $46 \%$ & $54 \%$ \\
\hline $\begin{array}{l}\text { Spécialistes en médecine } \\
\text { interne (1 à } 15 \text { ans } \\
\text { d'expérience) }\end{array}$ & $\begin{array}{l}\text { Penser à voix haute } \\
\qquad \\
\text { cartes conceptuelles }\end{array}$ & $\begin{array}{c}4 \text { problèmes cliniques complexes } \\
\text { simulés par écrit }\end{array}$ & $40 \%$ & $60 \%$ \\
\hline Spécialistes en néphrologie ${ }^{[11]}$ & $\begin{array}{c}\text { Penser à voix haute } \\
+ \\
\text { analyse différée sur } \\
\text { les verbatims } \\
\text { enregistrés }\end{array}$ & $\begin{array}{l}\text { Résoudre } 11 \text { problèmes } \\
\text { cliniques complexes }\end{array}$ & $63 \%$ & $37 \%$ \\
\hline $\begin{array}{l}\text { Spécialistes en gastro-entérologie } \\
\text { (plus de } 5 \text { ans d'expérience) }\end{array}$ & $\begin{array}{l}\text { Penser à voix haute } \\
\qquad+ \\
\text { analyse instantanée }\end{array}$ & $\begin{array}{c}4 \text { problèmes cliniques de } \\
\text { gastro-entérologie non complexes } \\
\text { (un seul diagnostic possible) }\end{array}$ & $94 \%$ & $6 \%$ \\
\hline
\end{tabular}

TRI : Taux de raisonnement inductif. TRD : Taux de raisonnement déductif. QROC : Question à réponse ouverte et courte. QCM : Question à choix multiple.

Nos résultats ne suggèrent pas de variations très franches des stratégies de raisonnement en fonction de l'expertise comme en témoignent les résultats du tableau I concernant le nombre de séquences déductives et le taux de raisonnement inductif. Le taux de raisonnement inductif semble diminuer progressivement avec l'expertise (respectivement 0,6 chez les étudiants de troisième année, 0,5 chez les étudiants de cinquième année et 0,4 chez les internistes). Ces résultats suggèrent, comme piste de recherche, une priorisation du raisonnement inductif lié à l'acquisition de savoirs théoriques en cours de deuxième cycle, tandis que le raisonnement déductif se développerait lors de l'acquisition de savoirs pratiques liés à l'expérience. Peu d'études comparent la fréquence de différentes stratégies de résolution de problème au sein du raisonnement médical diagnostique et la nôtre est la seule à se référer à des représentations cartographiques (cf. tableau II). Norman et al. ${ }^{[11]}$ ont retrouvé un taux global de raisonnement inductif comparable au nôtre de $63 \%$ chez des internes en médecine générale, médecine interne et des néphrologues ayant pour tâche de résoudre des problèmes cliniques complexes à voix 
haute mais ils ne constataient pas d'augmentation du recours au raisonnement déductif avec l'expertise puisque chez les experts toutes les inférences augmentaient, qu'elles soient inductives ou déductives. Heemskert et al. ${ }^{[5]}$ retrouvent un taux de raisonnement déductif chez des résidents en médecine interne de $54 \%$. Par ailleurs, le raisonnement apparaît plus « compilé » chez les experts si l'on en juge par les différences significatives observées en termes de sous-unités de raisonnement. Une plus grande compilation du discours associé au raisonnement avait déjà été montrée par Bordage et al. ${ }^{[25]}$.

En revanche, Coderré et al. ${ }^{[12,13]}$ montrent une prépondérance du raisonnement inductif chez les experts (jusqu'à 96 \%). Dans le cadre de leurs travaux, la résolution du problème était rendue explicite par des réponses à deux types de questions à choix multiples (QCM) comprenant soit moins de 5 options, soit plus de 10 options. Dans ces études deux facteurs étaient susceptibles de limiter le raisonnement déductif : d'une part l'effet d'indice (cueing effect) lié aux réponses proposées dans les QCM et, d'autre part, la nature de la tâche, qui s'avérait beaucoup moins complexe puisque les études concernaient des spécialistes en gastroentérologie, qui étaient confrontés à des problèmes de gastroentérologie avec un seul diagnostic possible.

Comme attendu, les problèmes $n^{\circ} 2$ et $n^{\circ} 4$ ont eu tendance à induire respectivement des séquences inductives et des séquences déductives. En revanche, le problème clinique $n^{\circ} 3$ n'a pas conduit à l'utilisation d'organigrammes décisionnels comme attendu. Trois explications peuvent être proposées : 1) le problème n'était pas suffisamment bien conçu pour favoriser l'utilisation d'un tel outil ; 2) la méthode d'analyse des cartes est inadaptée pour le repérage de ce type de séquence; 3) la méthode de représentation cartographique du raisonnement pendant que l'étudiant pense à voix haute est moins pertinente pour identifier ce type de séquence.

L'absence de prépondérance de la reconnaissance de forme pour le problème $n^{\circ} 1$ est probablement le fait d'une des limites de notre méthode. En effet, comme l'a montré Eva ${ }^{[26]}$, la reconnaissance de forme étant une stratégie de résolution de problème inconsciente, elle ne peut pas par conséquent être correctement identifiée par des protocoles basés sur l'analyse de la pensée à voix haute.

Ce travail en outre présente deux limites qu'il convient d'expliciter pour préciser le degré de validité externe des résultats :

- la reproductibilité de la méthode de représentation graphique du raisonnement par un tiers n'est pas rapportée dans cette étude mais a été étayée dans une étude antérieure montrant une reproductibilité inter-juge acceptable ${ }^{[27]}$.

- l'analyse est limitée par le caractère non authentique de la mise en situation diagnostique. Les participants avaient pour tache de résoudre un problème exposé par écrit où il était tacitement convenu que l'ensemble des symptômes était rapporté et qu'il n'était donc pas nécessaire de vérifier la présence ou l'absence d'autres signes, ce qui par définition ne pouvait que limiter le raisonnement déductif. Cette limite peut expliquer pourquoi il n'a pas été retrouvé de prépondérance du raisonnement hypothético-déductif chez les novices comme dans certaines études. La même analyse chez des étudiants raisonnant à voix haute en situation plus authentique (patients simulés par des acteurs ou d'autres étudiants par exemple) permettrait probablement de dégager plus nettement les stratégies déductives.

\section{Conclusion}

Ce travail exploratoire montre que l'utilisation d'une méthode originale de représentation cartographique $\mathrm{du}$ raisonnement clinique permet d'illustrer les principales stratégies de résolution de problème connues. Par ailleurs, l'étude du raisonnement diagnostique médical à partir de l'analyse structurelle de telles cartes procédurales retrouve une prépondérance des stratégies mixtes, tant chez les novices que chez les experts, lorsqu'ils sont confrontés à des problèmes cliniques complexes simulés par écrit.

En s'inscrivant dans la continuité des travaux publiés dans le domaine du raisonnement clinique 
diagnostique, les variations observées dans la structure du raisonnement en fonction du niveau d'expertise et du type du problème clinique plaident pour une certaine validité de cette méthode. L'utilisation de regroupements syndromiques et de catégories sont deux stratégies élaborées qui peuvent prendre toute leur place à coté des stratégies inductives déjà identifiées telles que la reconnaissance de script et le raisonnement basé sur des schémas décisionnels pré-établis.

\section{Contributions}

Pierre Pottier a contribué à la rédaction du protocole, la conduite de l'étude, l'analyse des données et rédigé le manuscrit. Bernard Planchon a contribué à la rédaction du protocole et à la conduite de l'étude. Jean-Benoît Hardouin a contribué à la rédaction du protocole, à la conduite de l'étude et à l'analyse des données. Véronique Sebille a participé à lecture et à la correction du manuscrit. Jean-Michel Rogez était garant de l'éthique du protocole. Jacques-Henri Barrier a participé à la rédaction du protocole, à la lecture et à la correction du manuscrit.

\section{ANNEXE}

\section{Problème clinique $n^{\circ} 1$}

Vous êtes appelé au chevet d'un enfant de 4 ans pour des difficultés à respirer évoluant depuis quelques heures, d'aggravation rapide. À votre arrivée, l'enfant est abattu, assis dans son lit. Le rythme respiratoire est à $28 \cdot \mathrm{min}^{-1}$. À l'inspiration, pendant laquelle l'enfant est particulièrement gêné, vous entendez un bruit aigu. La température est à $39^{\circ} 5$. À l'inspection de l'enfant, vous notez une impossibilité d'avaler la salive qui s'écoule par la bouche et un tirage sus-claviculaire.

\section{Problème clinique $n^{\circ} 2$}

Monsieur G, 65 ans est amené par sa femme pour des troubles du comportement d'aggravation progressive depuis 2 mois. Sa femme rapporte en effet une agressivité croissante envers elle, une tendance à oublier les faits récents et des épisodes de plus en plus fréquents pendant lesquels «il ne sait plus où il est».

À l'interrogatoire, Monsieur G est effectivement désorienté dans le temps et dans l'espace. Le MMS est à $18 / 30$.

À l'examen physique vous notez,

- $\mathrm{TA}=13 / 7$, pouls régulier à $80 \cdot \mathrm{min}^{-1}$, température à $37^{\circ} 5$.

- un état de vigilance normal mais des troubles de l'attention et une amnésie portant sur les faits récents.

- des réflexes vifs et diffusés aux membres inférieurs avec un signe de Babinski bilatéral.

- une hypopallesthésie bilatérale et symétrique, nette aux membres inférieurs.

- une diminution de la sensibilité tactile, une conservation de la sensibilité à la douleur aux membres inférieurs sans niveau sensitif.

- un subictère conjonctival.

- une pâleur cutanée.

\section{Problème clinique $n^{\circ} 3$}

Un homme de 67 ans, ancien éthylique, ne consommant actuellement plus d'alcool, est hospitalisé pour le bilan d'un ictère apparu sans douleurs ni fièvre. Dans ses antécédents, on note également une affection coronaire traitée par diltiazem (Tildiem). L'ictère est apparu de façon très progressive et évolue en s'accentuant de jour en jour. Il est indolore, apyrétique et s'accompagne depuis peu d'un prurit localisé aux mains. L'amaigrissement depuis un mois se chiffre à 3 kilogrammes. L'examen physique objective une vésicule tendue, facilement palpable. Les premiers résultats biologiques mettent en évidence une hyperbilirubinémie conjuguée très élevée à 520 micromol. $1^{-1}$, des phosphotases alcalines à 600 UI, transaminases normales, un taux de prothrombine à $45 \%$, un bilan électrolytique sanguin et urinaire normal. La numération formule montre : hémoglobine $9.5 \mathrm{~g} / 100 \mathrm{ml}$, VGM $70 \mu 3$. 


\section{Problème clinique $n^{\circ} 4$}

Margaret, 75 ans, vivant seule à domicile, se plaint d'un prurit nocturne et diurne, diffus, évoluant depuis plusieurs mois et rebelle aux traitements locaux (dont le bufexamac) et aux antihistaminiques. Il ne s'accompagne d'aucun signe général. L'examen dermatologique ne montre pas de lésion spécifique, en dehors d'une xérose importante et de discrètes lésions de grattage. On note dans ses antécédents :

- une fracture du col fémoral il y a 3 mois avec séjour en maison de repos ;

- des tassements vertébraux L1-L2, L2-L3 il y a 7 mois :

- un diabète de type 2 depuis 20 ans ;

- une hypertension artérielle ;

- un syndrome anxiodépressif.

Son traitement est le suivant : énalapril, metformine, paroxétine, bromazépam, paracétamol codéine.

Consigne : "résolvez ce problème diagnostique sachant que plusieurs hypothèses peuvent rester plausibles au terme de l'analyse »

\section{Références}

1. Yates JF, Tschirthart MD. Chapter 24: Decision-making. In: Eltovich P, Hoffman P (eds). The cambridge handbook of expertise and expert performance. Cambridge: New York, Cambridge University Press, 2006.

2. Eva KW. Ce que tout enseignant devrait savoir concernant le raisonnement clinique. Pédagogie Médicale 2005;6:225-34.

3. Fabre M. Situations-problèmes et savoir scolaire. Presse Universitaire de France 1999.

4. Nendaz M, Charlin B, Leblanc V, Bordage G. Le raisonnement clinique : données issues de la recherche et implications pour l'enseignement. Pédagogie Médicale 2005;6:235-54.

5. Heemskerk L, Norman GR, Chou S, Mintz M, Mandin H, McLaughlin K. The effect of question format and task difficulty on reasoning strategies and diagnostic performance in internal medicine residents. Adv Health Sci Educ Theory Pract, 2007 [On-line].

Disponible sur : www.springerlink.com/content/ g7w6026132pp2822/
6. Norman GR, Young M, Brooks L. Non-analytical models of clinical reasoning: the role of experience. Med Educ 2007;41:1140-5.

7. Mandin H, Jones A, Woloschuck W, Harasym P. Helping students learn to think like experts when solving clinical problems. Acad Med 1997;72:173-9.

8. Elstein AS, Schulman LS, Sprafka SA. Medical problem solving: an analysis of clinical reasoning. Cambridge (MA): Harvard University Press, 1978.

9. Charlin B, Boshuizen HPA, Custers EJ, Feltovich PJ. Scripts and clinical reasoning. Med Educ 2007:41:117884.

10. Katalunga-Moruzi C, Brooks LR, Norman GR. Coordination of analytic and similarity-based processing strategies and expertise in dermatological diagnosis. Teach Learn Med 2001;13:110-6.

11. Norman GR, Trott AD, Brooks LR, Smith EKM. Cognitive differences in clinical reasoning related to postgraduate training. Teach Learn Med 1994;6:114-20.

12. Coderre S, Mandin H, Harasym PH, Fick GH. Diagnostic reasoning strategies and diagnostic success. Med Educ 2003;37:695-703.

13. Coderre SP, Harasym P, Mandin H, Fick G. The impact of two multiple-choice question formats on the problemsolving strategies used by novices and experts. BMC Med Educ, 2004 [On-line]. Disponible sur : www . biomedcentral.com/1472-6920/4/23

14. Charlin B, Bordage G, Van Der Vleuten C. L'évaluation du raisonnement clinique. Pédagogie Médicale 2003;4:4252.

15. Fournier JP, Thiercelin D, Pulcini C, Alunni-Perret V, Gilbert E, Minguet JM et al. Évaluation du raisonnement clinique en médecine d'urgence : les tests de concordance de scripts décèlent mieux l'expérience clinique que les questions à choix multiples à contexte riche. Pédagogie Médicale 2006;7:20-30.

16. McLaughlin K, Coderre S, Mortis G, Fick G, Mandin H. Can concept sorting provide a reliable, valid and sensitive measure of medical knowledge structure? Adv Health Sci Educ Theory Pract 2007;12:265-78.

17. Marchand C, d'Ivernois JF. Les cartes conceptuelles dans les formations en santé. Pédagogie Médicale 2004;5:230-40.

18. Ruiz-Primo MA, Shavelson RJ. Problems and issues in the use of concepts maps in science assessment. J Res Sci Teach 1996;33:569-600.

19. West DC, Park JK, Pomeroy JR, Sandoval J. Concept mapping assessment in medical education: a comparison of two scoring systems. Med Educ 2002;36:820-6. 
20. King PM, Kitchener KS. Developping reflective judgment: Understanding and promoting intellectual growth and critical thinking in adolescents and adults. San Francisco (CA): Jossey-Bass, 1994.

21. West DC, Pomeroy JR, Park JK, Gerstenberger EA, Sandoval J. Critical Thinking in graduate medical education. A role for concept mapping assessment? JAMA 2000;284:1105-10.

22. Stensvold MS, Wilson JT. The interaction of verbal ability with concept mapping in learning from a chemistry laboratory activity. Sci Educ 1990;74:473-80.

23. Schmid RF, Telaro G. Concept mapping as an instructionnal strategy for high school biology. J Educ Res 1990; 84:78-85.

24. Tardiff J. L'évaluation des compétences. Documenter le parcours de développement. Chenelière Education, 2006.
25. Bordage G. Elaborated knowledge: a key to successsful diagnostic thinking. Acad Med 1994;69:883-5.

26. Eva KW, Brooks LR, Norman GR. Forward reasoning as a hallmark of expertise in Medicine: logical, psychological, and phenomenological inconsistencies. In: Shohov, SP, eds. Advances in Psychological Research, vol 8. New York: Nova Scotia 2002:42.

27. Pottier P, Hardouin JB, Hodges BD, Pistorius MA, Connault J, Durant C, Clairand R, Sebille V, Barrier JH, Planchon B. Exploring how students think: a new method combining think-aloud and concept mapping protocols. Med Educ 2010;44:926-35.

Correspondance et offprints : Pierre Pottier, Service de Médecine Interne A, Hôtel Dieu, CHU Nantes, 44093 Nantes Cedex 1, France.

Mailto : pierre.pottier@univ-nantes.fr 